\title{
Electrical Engineering and Electronics Teaching Aiming for Excellence Engineer Program
}

\author{
Hao Wang ${ }^{1}$ Qincui Fu ${ }^{2}$ \\ ${ }^{1}$ School of Economics and Management, East China Jiaotong University, Nanchang, China \\ ${ }^{2}$ School of Electrical and Electronic Engineering, East China Jiaotong University, Nanchang, China
}

\begin{abstract}
Electrical Engineering and Electronics is an important basic course for the non-electrical specialties of science and technology disciplines. It plays an important role in cultivating students' engineering practice ability. In order to accompany the implementation of Excellence Engineer Program and to meet the future oriented engineering industry's demand for high-technology and creative talents, curriculum innovation and teaching reform are carried out. Aiming at Excellence Engineer Program, in the process of construction of Electrical Engineering and Electronics course, research on innovative design for teaching and experiment is carried on in our school from the aspects of curriculum content, teaching model, experimental procedures.
\end{abstract}

Keywords: Electrical Engineering and Electronics, Excellence Engineer Program, teaching reform

\section{Introduction}

In China, restricted by the traditional concept and funds input, the patterns of engineering education of colleges and universities converged with those of science education. Since the enrollment expansion, the practice content of engineering education has three trends: authentication, mechanistic, unification. The engineering education lacks of the cultivation of abilities of autonomy, design, innovation. The training mode of emphasizing theory and neglecting practice results in the cycle phenomenon that enterprises have a severe shortage of technology, practical talents, while the employment rate of university graduates is more and more low. It causes the engineering leading talents are very scarce. This has become the critical factor restricting the industrialization process in our country.

Facing the urgent requirement of the development and upgrading of Chinese economy and the overall enhancement of international competitiveness, to foster a large number of engineering and technical talents of innovative ability, adapting to the needs of economic and social development of our country, the Ministry of Education proposed the Excellent Engineer Education Program (hereinafter referred to as Excellence Engineer Program). The implementation of the program marked the start of the quality project of engineering education. Its goal is to foster various high-quality engineering and technical talents of innovative ability and adapting to the needs of economic and social development, to serve for taking a new road to industrialization, building an innovation oriented country, fulfilling the strategy of talents state. In 2012, our school's mechanical design and manufacturing specialty, vehicle engineering specialty, communication engineering specialty were approved the second batch of "Excellent Engineer Education Program" pilot specialty.

Electrical Engineering and Electronics is an important basic course for the non-electrical specialties of science and technology disciplines, including the basic knowledge and its application in the various disciplines of strong, weak electricity. The course has the characteristics of wide range of knowledge, strong practicality and fast knowledge update. In order to accompany the implementation of Excellence Engineer Program and to meet the future oriented engineering industry's demand for high-technology and creative talents, curriculum innovation and teaching reform are carried out. The teaching reform not only can help students master the basic theory, knowledge and skills in electrical and electronic technology, but also is beneficial to training students' practical ability and creative ability, improve the engineering specialty students' engineering consciousness, engineering quality and engineering practice ability, lay a good foundation for future study and application of a deeper, more professional knowledge and skills.

Aiming at Excellence Engineer Program, in the process of construction of Electrical Engineering and Electronics course, research on innovative design for teaching and experiment is carried on in our school from the aspects of curriculum content, teaching model, experimental procedures.

\section{Reform of Course Contents and Teaching Mode}

Today, in the implementation of Excellence Engineer Program, Electrical Engineering and Electronics, as a technical basic course of engineering non-electrical majors, its teaching focus is not to let students grasp the deep theory, but lies in the cultivation of students' ability of applying the knowledge in electrical and electronic technology to their respective professional and innovative development in their respective professional. Therefore, the design of the course must be considered to build a complete and plastic knowledge framework for the students, but also from the engineering application point of view, to establish two-way and interact between theoretical knowledge and practical application.

The course contents of Electrical Engineering and Electronics can be divided into three levels. The first level refers to the basic theory, the concept and principle of electrical and electronic, these contents have 
independent chapters according to the theoretical system. The second level refers to the related knowledge necessary for the application of theory in engineering practice, for example, low voltage electrical appliances and electrical automatic control technology, sensor and automatic detection technology, analog to digital and digital to analog conversion etc., which plays a role of bridge from theory to practice. The third level is the training of general scientific method and specific research methods of electrical and electronic.

The teaching contents belong to the first level, in teaching need detail derivation. The teaching contents belong to the second level, focusing on application, in teaching need to design preview problems, guiding students to access to information and self-study.

In the third level, through the inquiry teaching mode, learning and research blending as a whole, for the purpose of cultivating the ability and improving thinking, engineering case teaching method is tried out, the typical teaching cases which have practical value in engineering application are designed, the teaching contents with engineering application background are supplemented. In the process of teaching, the first level and the second one in the curriculum system consist of the blending and progressive relation, focusing on training students' ability of scientific analysis and insight. Design of engineering case should consider the teaching needs for each professional, as well as the current development trend of different disciplines synthesizing, crossing and infiltrating, and focus on the introduction of basic concepts from different disciplines in the analysis of examples, expound the use of basic principle of electrical and electronics in every discipline.

\section{Reform of Experimental Teaching}

Electrical Engineering and Electronics has the characteristic of practice. Strengthening the laboratory construction and attaching importance to practice is always the most important aspects in the course construction. Aiming at Excellence Engineer Program, we try to reform the content system of experimental teaching, experimental conditions and experimental mode, increase the virtual experiment, opening experiment, comprehensive experiment, small system production, to vigorously increase the students' interest in study, experimental skills and application ability of electrical and electronic technology and cultivate innovation spirit.

\subsection{Introduction of EDA Technology and Use of Ad- vanced Experimental Teaching Means}

By introducing the EDA software into the whole process of teaching and experiment, helping students realize the construction of the virtual electronic laboratory, students can use EDA software to simulate the actual working process of various circuits in learning theory. Take Multisim of NI Company for example, students can input the circuit diagram, choose a variety of virtual signal generator as the outside excitation, observe the circuit working process, the output waveform and the quantitative indica- tors by the virtual instrument such as multi-meter, oscilloscope. The simulation result is near the actual situation of the physical circuit, very suitable for emulation and debugging in the courses such as Circuit Analysis, Ana$\log$ Circuit and Digital Circuit. Before the actual operation, students can preview the whole experiment process through the EDA software, and in the actual operation, put into practice with the physical circuit and instruments and equipment. The experimental teaching model of theory teaching, computer simulation and putting into practice can not only stimulate students' interest in learning and make students master the application method of modern EDA technology, but also enhance the application and practical ability and give students free space. And then students can target any application, design all kinds of circuits to observe their work condition, to find out the difference of circuits. The essence of the experimental teaching model is a kind of cultivation mode of independent learning, as an old saying "good delegate to the fish, it is better to delegate to fish".

\subsection{Strengthen the Reform of Experimental Con- tents and Method and Cultivate Students' Com- prehensive Design Ability}

In the experimental contents, the verification experiments are properly compressed, at the same time designing and comprehensive experiments are strengthened. Emphasis is placed on the cultivation of comprehensive application ability and innovation ability of students. Some verification experiments which have the characteristics of basic teaching selected, the student can deepen the understanding of theory content and also exercise the use of instrumentation. On this basis, the comprehensive and design experiments are strengthened, such as the AC motor control experiment can be extended to design and synthesis. In designing comprehensive experiments, in order to avoid students' blindness in the process of experiment and ensure the security of experiments, modern teaching methods of EDA technology should be made full use of. Students are required to give EDA simulation results of the designed circuit before actual operation, which are approved by the teacher later, and then can begin to experiment. In the comprehensive experiment, students are allowed to group by themselves and not strictly limited operation time. There are the following two methods: the one is that the teacher design some comprehensive subject, let students choose the topic and then complete the design; the other is that students design subject by themselves, teacher audit after. Only in this way can we combine theory and practice, enhance the interest of students, cultivate students' ability of using the knowledge to analysis and solve the question and innovation ability. In the process of experimental teaching, we pay attention to the heuristic teaching method, change the previous method of explaining the experimental process and steps in detail. By using heuristic teaching, teachers guide the students to do experiments, that is, only tell about matters needing attention, less about specific contents, especially in the design experiments of some high level. In order to cultivate students' self-study ability, teachers do not repeat the 
explanation of the basic knowledge learned before, just according to the design task put forward references, let students self-study. At the same time, adopting the method of combining collective guidance with individual question-and- answer, the tutor guides students to consult the manuals, diagrams and literatures according to the topic. In addition, in the course of the experiment, the circuit do not work correctly is one of the most common fault, the tutor need not directly point out the cause of the fault, but to remind students to use what has been learned to solve their problems independently.

\subsection{Using Resources of Research Laboratory to Serve for Undergraduate Practice Teaching}

At present many colleges and universities propose the transition from teaching to teaching-and-research, and research laboratories in accordance with past routine, generally is used for scientific research, only open to graduates. Due to science and technology develop more and more quickly and the update cycle of experimental equipment is short, the utilization rate of some equipment is very low. While to the undergraduates, this equipment is very superior, but not used, it is also a waste of resources. Work in the research laboratory has the obvious characteristics of practicality, innovation, which fully meets Excellence Engineer Program's requirements for undergraduate practical teaching. Therefore, the school should afford policy and financial support, expand the scale of the laboratory, transform research laboratory into teaching-and-research laboratory. Undergraduate students enter the laboratory, engaged in some simple basic work, with the ability to improve, and then participate into more high level research. Because the research laboratory has the characteristics of preciseness and innovation, students in the research work can inherit the spirit of practice and innovation from the mentor and senior there and get enlightenment. At the same time, the organization and management of research laboratory is similar to enterprise management, therefore it can develop students' teamwork spirit, make them indirectly learn contemporary enterprise culture and management culture, lay good foundation for the future after graduation quickly into social, which is not the general teaching process can train and get.

\subsection{Increase Opening-up of the Laboratory and Im- prove Utilization Rate of the Laboratory.}

In the reform of experimental teaching methods and means, one important link is the open-up laboratory, which guarantees students of fulfilling design and comprehensive experiment. The open laboratory provides students more experimental resources and experimental sites, offers students of poor experimental skills two hands-on opportunities and lets students of strong experimental skills obtain the sublimation in practice. Students do comprehensive experiment designed by their own make them change from being arranged by the tutor passively into designing and implementing actively, cultivating students' comprehensive quality.

\section{Conclusions}

Combining with teaching of Excellence Engineer Program pilot class, our school make the enhancement of students' self-learning ability, engineering quality and innovation consciousness as the primary goal of the reform of the Electrical Engineering and Electronics course, through the reform and innovation of curriculum content, teaching model and experimental procedures, promote the development of Inquiry-based teaching model, implement Excellence Engineer Program by the reform of the Electrical Engineering and Electronics course's teaching and experiment.

\section{Acknowledgements}

This research was supported by Jiangxi Province Educational Department Teaching Reform and Research Project (No.JXJG-12-5-23) and Scientific Research Foundation of East China Jiaotong University (No.11DQ08).

\section{References}

[1] Jian Lin, “Outstanding Engineers' Innovation Ability Training" [J]. Research in Higher Education of Engineering, 2012(5): 1-17.

[2] Ning Lu, "Research on the Reform of Electrotechnics Teaching Based on Excellence Engineer Training" [J]. Education and Vocation, 2014(15): 136-137.

[3] Anfu Zhang, Xingfeng Liu, "Reflection on the Implementation of 'A plan for Educating and Training Outstanding Engineers"' [J]. Research in Higher Education of Engineering, 2010(4):56-59. 